\title{
Sexualidade na terceira idade: a visão dos idosos em um município do interior do estado de São Paulo
}

\author{
Sexuality in the third age: a vision of the elderly in a municipality of the interior of the \\ state of São Paulo \\ Sexualidad en la tercera edad: una visión de los ancianos en un municipio del \\ interior del estado de São Paulo \\ Luiz Fernando de Andrade Silva1*, Adriana Avanzi Marques Pinto.
}

\begin{abstract}
RESUMO
Objetivo: Pretende-se com esse estudo descrever o perfil de vida e sexual dos idosos, de um município do interior paulista e analisar seu conhecimento sobre sexualidade. Métodos: Foi aplicado um questionário já validado pelo Laboratório de Gênero, Sexualidade e Corporeidade (LAGESC/UDESC), composto por 7 perguntas. Para as perguntas de número 1, 2, 5 e 7 foi utilizado uma escala com variação de 0 a 10, que corresponde a nada (0), pouco (1 a 3), medianamente (4 a 6), razoavelmente (7 a 9) e muito (10). Resultados: Participaram do estudo 49 idosos que ao serem questionados sobre a importância do sexo na juventude, 33\% disseram ser razoavelmente importante e 53\% muito importante. Em relação a frequência sexual atual $44 \%$ dos entrevistados responderam 1 vez por semana. Conclusão: $O$ fator frequência sexual acaba não sendo o principal para satisfação, pois os idosos falam muito sobre carinho, companheirismo e atenção. Vale ressaltar a importância da sexualidade para eles e a importância da melhora da abordagem pelos profissionais de saúde.
\end{abstract}

Palavras-Chave: Envelhecimento, Sexualidade, Saúde do Idoso.

\begin{abstract}
Objective: This study aims to describe the life and sexual profile of the elderly in a city in the interior of São Paulo and analyze their knowledge about sexuality. Methods: A questionnaire already validated by the Gender, Sexuality and Corporeity Laboratory (LAGESC / UDESC), was composed of 7 questions. For questions 1, 2, 5 and 7 a scale ranging from 0 to 10 was used, which corresponds to nothing (0), little (1 to 3), medium (4 to 6), reasonably (7 to 9) and much (10). Results: Participating in the study were 49 elderly people who, when asked about the importance of sex in youth, $33 \%$ said to be reasonably important and 53\% very important. Regarding the current sexual frequency, 44\% of respondents answered once a week. Conclusion: The sexual frequency factor is not the main factor for satisfaction, since the elderly talk about affection, companionship and attention. It is worth emphasizing the importance of sexuality for them and the importance of improving the approach by health professionals.
\end{abstract}

Key words: Aging, Sexuality, Health of the eldery.

\section{RESUMEN}

Objetivo: Se pretende con este estudio describir el perfil de vida y sexual de los ancianos, de un municipio del interior paulista y analizar su conocimiento sobre sexualidad. Métodos: Se aplicó un cuestionario ya validado por el Laboratorio de Género, Sexualidad y Corporeidad (LAGESC / UDESC), compuesto por 7 preguntas. Para las preguntas de número 1, 2, 5 y 7 se utilizó una escala con variación de 0 a 10 , que corresponde a nada (0), poco ( 1 a 3), medianamente (4 a 6), razonablemente ( 7 a 9 ) y mucho

${ }^{1}$ Fundação Educacional do Município de Assis (FEMA), Assis - SP. *E-mail: luizfas91@gmail.com

SUBMETIDO EM: 1/2019

ACEITO EM: 2/2019

PUBLICADO EM: 5/2019

REAS/EJCH | Vol. 11 (10) | e304 | DOI: https://doi.org/10.25248/reas.e304.2019 Página 1 de 5 
(10). Resultados: En el estudio participaron 49 ancianos que, al ser cuestionados sobre la importancia del sexo en la juventud, el 33\% dijo que era razonablemente importante y un 53\% muy importante. En relación a la frecuencia sexual actual el $44 \%$ de los entrevistados respondieron 1 vez por semana. Conclusión: El factor frecuencia sexual termina no siendo el principal para satisfacción, pues los ancianos hablan mucho sobre cariño, compañerismo y atención. Es importante resaltar la importancia de la sexualidad para ellos y la importancia de la mejora del abordaje por los profesionales de salud.

Palabras clave: Envejecimiento, La sexualidad, Salud de los ancianos.

\section{INTRODUÇÃO}

O processo de envelhecimento é constante em nossa vida, lidamos com isso todos os dias. A velhice é uma etapa da vida ainda bastante significativa, na qual o idoso(a), muitas vezes, trabalha de forma ativa, prática esportes e tem uma vida sexual regular. Ainda é uma etapa de procura do prazer, da felicidade, do bem-estar físico, psíquico e mental (MINISTÉRIO DA SAÚDE, 2006).

Ao buscar a definição de idoso, tem-se de acordo com o Estatuto do Idoso (2013), que idosos são aqueles com idade igual ou superior a 60 (sessenta) anos. De acordo com o Instituto Brasileiro de Geografia e Estatística (IBGE), há aproximadamente uma população de 20 milhões de pessoas com 60 ou mais e isso representa $10 \%$ da população total no país. Segundo a Organização Mundial da Saúde (OMS), até 2025 haverá um aumento de 15 vezes em relação ao número de idosos, o que representa, em números, cerca de 32 milhões de pessoas com 60 anos ou mais. Devido ao intenso crescimento da população idosa, o Brasil ocupará, em 2025 o sexto lugar na lista dos países com a maior quantidade de idosos (MINISTÉRIO DA SAÚDE, 2010).

Quando falamos em sexualidade, aqueles com melhores condições de saúde continuarão as suas atividades sexuais que são de extrema importância. Quando feito de forma desprotegida, muito idosos estão expostos à DSTS/HIV mesmo que não acreditando ser possível nessa fase da vida. Entre os anos de 2002 a 2014 foram diagnosticados no brasil 17.861 casos de AIDS em pessoas com 60 anos ou mais (CASSÉTTE JB, et al., 2016). Contudo, além dos números de idosos diagnosticados com HIV, é importante entender o porquê de não se sentirem vulneráveis e como esses idosos entendem sexualidade.

O idoso apresenta uma visão ampla da sexualidade, que compreende o toque, troca de olhares, carinho, companheirismo, valores, crenças, comportamento, desejo, culturais e tantos outros fatores que influenciam diretamente a forma com que esses idosos vivenciam a sexualidade. Para os idosos, sexualidade é um tema mais dinâmico do que conseguimos imaginar, mesmo que para a sociedade o tema sexualidade na terceira idade seja visto com preconceitos e ideias erroneamente preconcebidas (VIEIRA S, et al., 2014).

Um dos grandes problemas enfrentado pelos idosos, relacionado à sexualidade, é a família. Muitos familiares julgam, opinam e quase sempre fazem escolhas pelos idoso. Com isso, muitos idosos acabam por perder o poder de escolha e sua autonomia, não apenas quando falamos de sexualidade. (CATUSSO MC, 2005). Outro fator importante é o preconceito perante a sociedade, que tem a ideia de idoso assexuado, como se o ato sexual fosse errado ou inapropriado nessa fase da vida. Porém, não existem motivos para um idoso saudável não manter uma vida sexual de forma protegida e ativa (VIEIRA KFL, et al., 2016).

Outro problema encontrado pelos idosos é relacionado as alterações do corpo na velhice. Homens e mulheres idosos passam por importantes alterações fisiológicas e hormonais que podem ou não alterar o desejo sexual. Nos homens podemos observar alterações como: pênis com ereção prejudicada, tempo maior para o orgasmo, ejaculação prejudicada e redução do líquido seminal (ALENCAR DL, et al., 2004).

Já nas mulheres, as alterações começam um pouco mais cedo, na menopausa com redução da produção de hormônios. Apresentam também, pele seca e mais fina, lubrificação da vagina diminuída, um dos pontos que mais preocupam as mulheres idosas, que, por esse motivo não levam uma vida 
sexualmente ativa com receio da dor causada pela pouca lubrificação natural da vagina. A masturbação passa a ser uma opção menos dolorosa quando não há parceiro. Assim, o sexo vaginal deixa de ser a única fonte de prazer e as idosas descobrem outras formas de sentirem prazer nas regiões erógenas (ALENCAR DL, et al., 2004). Com esta pesquisa conhecemos o perfil da sexualidade dos idosos do município de Assis-SP, descrevemos o perfil do idoso com vida sexual ativa, o perfil de vida sexual do idoso e analisamos o conhecimento dos idosos de Assis sobre sexualidade.

\section{MÉTODOS}

Trata-se de uma pesquisa de campo de abordagem quantitativa sobre sexualidade na terceira idade em um município do interior paulista. Foi aplicado um questionário já validado pelo Laboratório de Gênero, Sexualidade e Corporeidade (LA-GESC/UDESC), composto por 7 perguntas, sendo que para as perguntas 1, 2, 5 e 7 foi utilizado uma escala com variação de 0 a 10, que corresponde a nada (0), pouco (1 a 3), medianamente (4 a 6), razoavelmente ( 7 a 9) e muito (10) sendo as demais de resposta aberta. A escolha dos participantes foi aleatória e voluntária, sendo o local para realização do convite a sala de espera das Unidades Básicas de saúde, Unidades de Saúde da Família e o Clube da terceira idade da cidade. A coleta de dados se encerrou em 2 meses (abril a maio de 2017), por se tratar de um projeto de iniciação científica. Em relação aos aspectos éticos, o estudo foi aprovado no Comitê de Ética sob o CAAE 62922816.7.0000.5406. Após a coleta os dados foram tabulados e analisados para responder os objetivos desta pesquisa. Para análise quantitativa foi realizado cálculos de porcentagem simples para representação das escolhas apontadas pelos entrevistados sobre a sexualidade e como eles lidam com essas questões frente às mudanças que sofrem por conta da idade.

\section{RESULTADOS E DISCUSSÕES}

O total de idosos do município é de 13.442, no total esse estudo contou com a participação de 49 idosos, que apresentaram uma média de idade de 75 anos (IBGE, 2010), quando questionado sobre a importância do sexo na juventude, 33\% disseram ser razoavelmente importante e 53\% muito importante. Quando questionados sobre a satisfação sexual atual, $21 \%$ disseram estar não estarem satisfeitos, $25 \%$ razoavelmente e apenas $20 \%$ muito satisfeito. Sobre se considerar sexualmente ativo atualmente, $20 \%$ se diz não ativo, 25\% razoavelmente ativo e $20 \%$ ativo. Sobre a importância atual do sexo, 17 disseram não ser importante e $57 \%$ ser muito importante. Analisando a literatura percebemos que a maioria dos idosos considera o sexo importante para o bem-estar físico e psicológico, auxiliando na diminuição de patologias físicas e mentais relacionadas a idade avançada (OLIVEIRA LB, et al., 2015). 0 gráfico 1 ilustra os dados obtidos acerca da importância sexual na juventude e atualmente, sobre satisfação e importância atual do sexo e se considerarem sexualmente ativo.

Gráfico 1 - Importância e satisfação sexual na juventude e atualmente, Assis 2017.

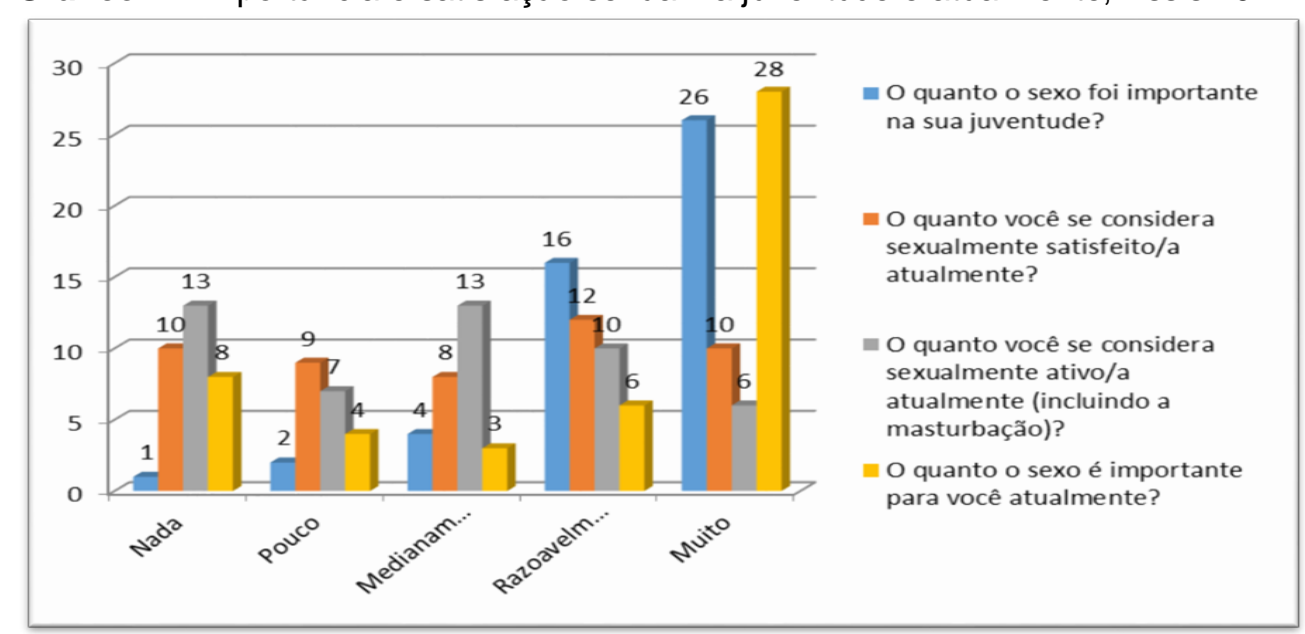

Fonte: Dados da pesquisa, 2017. 
A respeito da frequência sexual semanal na juventude, a maior variação foi de 3 vezes na semana (21\%). A maioria dos idosos na juventude não se sentiam preparados para iniciar a vida sexual, buscando informação com amigos. Nem todos que consideravam importante, praticavam o ato com maior frequência. Em relação a frequência sexual semanal atual, 35\% disseram nenhuma vez, $21 \%$ duas vezes e $44 \%$ responderam $1 \mathrm{vez}$. A literatura mostra que essa frequência realmente diminui, já que o idoso entende a sexualidade não como o ato sexual em si, mas como algo complexo, que envolve cumplicidade e carinho (VIEIRA KFL, et al., 2012). O gráfico a seguir ilustra os dados obtidos acerca da frequência sexual atual e na juventude.

Gráfico 2 - Frequência sexual na juventude e atual, Assis 2017.

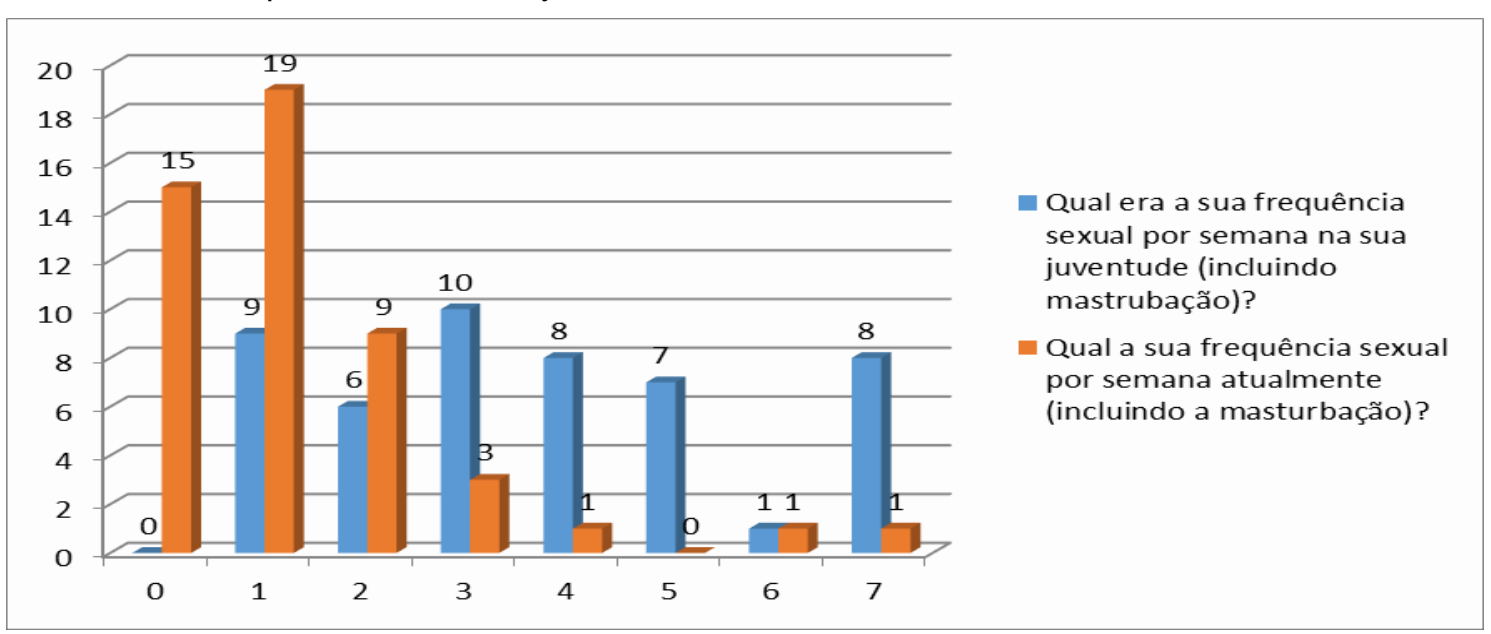

Fonte: Dados da pesquisa, 2017.

Em relação a parceria fixa, $57 \%$ responderam sim e $43 \%$ responderam não, sendo que $36 \%$ possuem marido/esposa e $64 \%$ namorado(a). Nesse período da vida, as pessoas não querem mais se sentir sozinhas ou fracas, por isso percebe-se aumento pela procurar de parceiros fixos ou não. Os idosos querem compartilhar pensamentos e ações, além de receberem ajuda dos parceiros. Na terceira idade, o namoro beneficia saúde mental e física, tudo melhora quando se sente amado. Essa parceria e a relação de cuidado para o idoso tem tanta valia quando a relação sexual. Para o idoso vivenciar um namoro significa ser feliz e ter mais vontade de viver, apesar dos impedimentos que a idade avançada traz. A terceira idade pode ser o momento perfeito para se mudar aspectos e conceitos da vida sexual (LAURENTINO NRS, et al., 2006).

Ao comparar os dados obtidos nesse estudo com o estudo realizado por Cardoso (2009), que utilizou o mesmo questionário em sua coleta de dados, percebe-se que existe uma relação entre os itens: maior frequência sexual atual, parceria fixa, ser sexualmente ativo e importância atual do sexo. Assim como Cardoso FL (2009), observa que, na juventude, no estudo citado para as mulheres, sentir-se sexualmente satisfeito estava relacionado com a importância do sexo naquele período de vida. Outro ponto importante é que, em ambos os trabalhos, as idosas com parceiro fixo acham o sexo importante, tem desejo e gostam do sexo, porém, o que difere é que não são todas que praticam o ato sexual em si devido a patologias próprias ou do parceiro.

Já em relação aos homens, observa-se semelhança na relação feita entre frequência sexual na juventude e importância e satisfação naquele período. Os resultados apontam tanto homens quanto mulheres não se consideram sexualmente ativos atualmente e a frequência sexual atual também é inferior que na juventude. Já Cardoso FL (2009) observa essa diferença apenas nos homens. Outro dado importante é que dos 49 idosos participantes, $57 \%$ dizem que o sexo é muito importante, porém a maior frequência sexual por semana atualmente foi de apenas 1 vez, com o total de $38,7 \%$ idosos, seguido por nenhuma vez com $30,6 \%$ idosos e $57 \%$ dos entrevistados possuem parceria fixa. Portanto, observa- se 
que o sexo é importante e ter parceiro fixo não é fator decisivo para a frequência sexual. Como Muitos idosos relataram, mesmo sentindo o desejo sexual, não conseguem praticar 0 ato em si devido à, por exemplo, cardiopatias graves.

Cardoso FL (2009), apresenta que a importância do sexo e a frequência sexual diminuem com a idade, contudo, observamos que para $57 \%$ participantes o sexo é muito importante e apenas a frequência diminui devido aos problemas já relacionados. Enfim, conclui-se que mesmo entre os idosos sexualmente ativos e que compreendem o sexo com grande importância, a frequência semanal diminui atingindo homens e mulheres de acordo com as particularidades de cada gênero.

\section{CONCLUSÃO}

A partir da aplicação do questionário e melhor conversa com os idosos, ficou claro a grande importância da sexualidade para eles, mesmo que não seja consumado o ato sexual em si, já que eles veem a sexualidade de uma maneira muito mais ampla. Contudo, muitos não e abrem com a família e profissionais de saúde por terem vergonha ou pouca abertura. Muitos profissionais da atenção primária sentem-se incomodados ou não veem importância de abordar esse tema com os idosos, tanto nas visitas domiciliares como em consultas na própria unidade. Porém, essa abordagem tanto das famílias como dos profissionais se faz necessária, principalmente em relação à quantidade de idosos com DST/AIDS, que só aumenta a cada ano. Para que essa porcentagem diminua e esses idosos tenham relação sexual mais segura e com qualidade, uma melhor abordagem pelos profissionais bem como educação dos idosos e seus familiares se faz muito importante. Sempre pensando na melhora da qualidade de vida dessa população.

\section{AGRADECIMENTOS}

À Secretaria de Saúde do Município de Assis e às Unidades Básicas de Saúde que possibilitaram a realização desta pesquisa.

\section{REFERÊNCIAS}

1. ALENCAR DL, et al. Fatores que interferem na sexualidade de idosos: uma revisão integrativa, Ciência e Saúde Coletiva. 2014;19(8):3533-42.

2. BRASIL. Lei federal $n=10.741$ de 03 de outubro de 2003. Brasília (DF); 2004.

3. MINISTÉRIO DA SAÚDE (BR). Atenção à saúde da pessoa idosa e envelhecimento. Brasília (DF);2010.

4. MINISTÉRIO DA SAÚDE (BR). Caderno de atenção básica - envelhecimento e saúde da pessoa idosa. Brasília (DF); 2006.

5. CASSÉTTE JB, et al. HIV/Aids em idosos: estigmas, trabalho e formação em saúde. Rev. Bras. Geriatr. Gerontol. 2016;19(5):733-44

6. Cardoso FL. Questionário/roteiro de entrevista sobre a sexualidade na terceira idade (QSTI). 2009. [Disponível em: http://www.cefid.udesc.br/laboratorios/lagesc/? modo=instrumentos-de-pesquisa. Acesso em: 14 out. 2017

7. CATUSSO MC. Rompendo o silêncio, desvelando a sexualidade em idosos. Revista Virtual Textos \& Contextos 2005;4(4):1-19.

8. INSTITUTO BRASILEIRO DE GEOGRAFIA E ESTATÍSTICA (BR). Perfil dos idosos responsáveis por domicílios no Brasil 1991. 2002. Disponível em: http://www.ibge.com.br/home/estatistica/populacao/perfilidoso/tabela1_1.shtm. Acesso em: 7 jul. 2016.

9. LAURENTINO NRS, et al. Namoro na terceira idade e o processo de ser saudável na velhice: recorte ilustrativo de um grupo de mulheres. Revista Brasileira de Ciências do Envelhecimento Humano. 2006;3(1):51-63.

10. OLIVEIRA LB, et al. Sexualidade e envelhecimento: avaliação do perfil sexual de idosos não institucionalizados. Rev. Ciên. Saúde Nova Esperança. 2015; 13(2):42-50.

11. VIEIRA KFL, et al. A Sexualidade na velhice: representações sociais de idosos frequentadores de um grupo de convivência. Psicologia: ciência e profissão.2016;36(1):196-209.

12. VIEIRA KFL, et al. Sexualidade na velhice: um estudo de representações sociais. Psicologia e saber social. 2012;1(1):12028.

13. VIEIRA S, et al. A vivência da sexualidade saudável nos idosos: O contributo do enfermeiro. Revista de Ciência da Saúde da ESSCVP. 2014; 6:36-45. 